International Journal of Pure and Applied Mathematics

Volume 100 No. 2 2015, 299-307

ISSN: 1311-8080 (printed version); ISSN: 1314-3395 (on-line version)

url: http://www.ijpam.eu

doi: http://dx.doi.org/10.12732/ijpam.v100i2.10

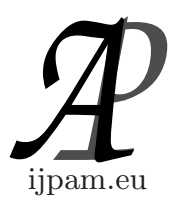

\title{
THE EVOLUTION OF AN ANISOTROPIC HYPERBOLIC SCHRÖDINGER MAP HEAT FLOW
}

\author{
P.H. Zhong \\ Department of Mathematics \\ Guangdong University of Education \\ Guangzhou, 510640, P.R. CHINA
}

\begin{abstract}
Solution of Schrödinger map heat flow equation with applied field in 2-dimensional $\mathcal{H}^{2}$ space is obtained. Two different methods are used to construct the norm -1 exact solution. The solution admit a finite time singularity or a global smooth property.
\end{abstract}

AMS Subject Classification: 35K10, 35K65, 35Q40, 35Q55

Key Words: Schrödinger map, hyperbolic, singularity, smooth solution

\section{Introduction}

In this paper, a class of generalized ferromagnetic material equation will be studied. We concentrate on the evolution of the vary solution of this equation. Ferromagnetic material equation which we usually refer to Landau-Lifhsitz equation (abbreviated LLE) is the kernel equation of the ferromagnetic materials. Combining the predecessors' research results, L.D. Landau and E.M. Lifshitz propose the dispersion equations of the magnetization in 1935 . Dynamics of Magnetic domain wall described by LLE exhibits very important applications in materials science. It is the fundamental equation describing the evolution of the density of magnetic ferromagnetic material. In fact, LLE exhibits the 
different properties in various target manifold. In this paper, we explore the evolution of LLE on the target manifold $\mathcal{H}^{2}$. Because complete LLE with the variety of external field is too complex, this study concentrates only on the anisotropic hyperbolic LLE without Gilbert damping term:

$$
S_{t}=S \dot{\wedge}(\triangle S)+S \dot{\wedge}(S L),
$$

where $S=\left(s_{1}, s_{2}, s_{3}\right)$ is spin vector. $\dot{\wedge}$ denotes the pseudo cross product. Exactly, $\vec{a} \dot{\wedge} \vec{b}=(\vec{a} \wedge \vec{b}) \operatorname{diag}\{1,1,-1\} . \quad|S|^{2}=s_{3}^{2}-s_{1}^{2}-s_{2}^{2}=1, s_{3}>0$ in hyperbolic space $\mathcal{H}^{2}$. Spin vector $S=\left(s_{1}, s_{2}, s_{3}\right) \in \mathcal{H}^{2} \hookrightarrow R^{2+1}$. Magnetic anisotropy $L=\operatorname{diag}\{0,0, \lambda\}$ where $\lambda$ is a constant.

More clearly, we all call (1) the anisotropic hyperbolic Schrödinger map heat flow. Schödinger map heat flow (SMF for short) is an important equation of differential geometry and physics. Usually, SMF can be regards as a foundation form of LLE. Hence, the study of the evolution of SMF is helpful for understanding the property of the ferromagnetic materials. In fact, if we define the mapping $S: R^{3} \times R \rightarrow(M, h, J)$ where $h$ and $J$ are the metric and the complex structure on Kähler manifold respectively, SMF with the application field is defined as follows

$$
\frac{\partial S}{\partial t}=J \sum_{l=1}^{n} D^{l} \partial_{l} S+J(S L),
$$

where $D$ denotes the covariant derivative of $S^{-1} T M$.

In (2), if we use $\mathcal{H}^{2}$ and $\dot{\Lambda}$ represent the manifold and complex structure, (2) and (1) are equal to each other. Otherwise, if $M=\mathrm{S}^{2}$ and $J(S)=S \Lambda$, (2) is anisotropic Landau-Lifshitz equation. In this situation, (2) becomes

$$
\frac{\partial}{\partial t} S=S \wedge(\triangle S)+S \wedge(S L) .
$$

In recent 20 years, the evolution of various solutions (singularity and smooth) of SME and LLE have attracted a considerable amount of attention (see [1, 2, 3, $4,5,6,9,10,11])$. Especially, the authors propose the exact solutions of $(3)$ and construct the exact transformation matrix between isotropic and anisotropic LLE in $[7,8]$. But it is worth to pointing out: compared to the equation of the $S^{2}$ type, the research of (2) is still in its beginning time. Under the conditions of hyperbolic manifolds, the evolution of (2) is not clear. Can the solution forms a singularities in finite time, or the solution is global? Furthermore, can the energy of the solution be finite? It is worth to study these questions. In this paper, we study these question through some examples. 


\section{Finite Time Singularity Solution I}

By stereographic projection, the projection is from $\mathcal{H}^{2}$ to the extend complex plane $C_{\infty}$. Accordingly, (1) can be transformed into a derivative Schrödinger equation. Specifically, using the following conversion

$$
U=\frac{s_{1}+i s_{2}}{1-s_{3}}
$$

(1) change into the following nonlinear Schödinger equation:

$$
i U_{t}=-\triangle U-\frac{2 \bar{U}}{1-|U|^{2}} \sum_{j=1}^{n}\left(\partial_{j} U\right)^{2}+\lambda \frac{1+|U|^{2}}{1-|U|^{2}},
$$

where $\bar{U}$ stands for the conjugate complex numbers of $U$.

Right hand side of (5) contains a first derivative term. In the same time, the coefficient of if is nonlinear. Hence, (5) (or (1)) is hard to resolve due to these two characteristics. In fact, the solution of (5) is easy to obtained only in space dimension $n=1$. In this situation, (5) is essentially equivalent to a nonlinear Schrödinger equation without derivative term. If $n \geq 2,(5)$ is not a completely integrable system. It will be difficult to find out the traveling wave solution or non-traveling wave solution of (5). In this paper, we only study the solution of (5) in cylindrical coordinates. In this case, (5) can be transformed into

$$
i U_{t}=-U_{r r}-\frac{n-1}{r} U_{r}-\frac{2 \bar{U}}{1-|U|^{2}} U_{r}^{2}+\lambda \frac{1+|U|^{2}}{1-|U|^{2}} .
$$

In fact, (5) and (1) can be transformed between each other. If selected

$$
\left(s_{1}, s_{2}, s_{3}\right)=\left(\frac{ \pm 2 \operatorname{Re}(U)}{1-|U|^{2}}, \frac{ \pm 2 \operatorname{Im}(U)}{1-|U|^{2}}, \frac{1+|U|^{2}}{1-|U|^{2}}\right),
$$

(5) can be transformed into a (1). So, we can resolve (6) to obtain the solution of (1).

We selected the solution as the following special form

$$
U=e^{i\left(f(t) r^{a}+h(t)\right)} \mathrm{g}(t)
$$

where $r=\left(\sum_{j=1}^{n} x_{j}^{2}\right)^{1 / 2} ; f(t), h(t)$ and $g(t)$ function is to be determined; $a$ is some special constant. 
Substituting (8) into (6), then separating the real part and imaginary part, we get

$$
\begin{gathered}
-g(t)\left(\frac{\mathrm{d}}{\mathrm{d} t} f(t)\right) r^{a}-g(t) \frac{\mathrm{d}}{\mathrm{d} t} h(t)-\frac{(f(t))^{2}\left(r^{a}\right)^{2} a^{2} g(t)}{r^{2}} \\
-2 \frac{(g(t))^{3}(f(t))^{2}\left(r^{a}\right)^{2} a^{2}}{\left(1-(g(t))^{2}\right) r^{2}}-\frac{\lambda g(t)}{1-(g(t))^{2}}-\frac{\lambda(g(t))^{3}}{1-(g(t))^{2}}=0, \\
\frac{\mathrm{d}}{\mathrm{d} t} g(t)+\frac{f(t) r^{a} a g(t) n}{r^{2}}-2 \frac{f(t) r^{a} a g(t)}{r^{2}}+\frac{f(t) r^{a} a^{2} g(t)}{r^{2}}=0 .
\end{gathered}
$$

It is difficult to solve (9) and (10). However, if $a=n=2$, we obtain the following differential equations

$$
\left\{\begin{array}{l}
\frac{\mathrm{d}}{\mathrm{d} t} h(t)+\frac{\lambda}{1-(g(t))^{2}}+\frac{(g(t))^{2} \lambda}{1-(g(t))^{2}}=0 \\
g(t)\left(\frac{\mathrm{d}}{\mathrm{d} t} f(t)\right) r^{2}+4(f(t))^{2} r^{2} g(t)+8 \frac{(g(t))^{3}(f(t))^{2} r^{2}}{1-(g(t))^{2}}=0 \\
\frac{\mathrm{d}}{\mathrm{d} t} g(t)+4 f(t) g(t)=0 .
\end{array}\right.
$$

Solving (11), the exact solution of it is as follows

$$
\left\{\begin{array}{l}
f(t)= \pm \frac{C_{2}}{4 \sqrt{t^{2} C_{2}^{2}+2 t C_{2} C_{3}+C_{3}^{2}-4}}, \\
h(t)=\mp \frac{\lambda \sqrt{t^{2} C_{2}^{2}+2 t C_{2} C_{3}+C_{3}^{2}+4}-C_{1} C_{2}}{C_{2}}, \\
g(t)=\frac{1}{2}\left(C_{2} t+C_{3} \mp \sqrt{t^{2} C_{2}^{2}+2 t C_{2} C_{3}+C_{3}^{2}-4}\right),
\end{array}\right.
$$

where $C_{1}, C_{2}$ and $C_{3}$ are constants.

According to (8) and (12), it will not difficult to find out the solution of (6)

$$
\begin{aligned}
U= & \mathrm{e}^{\frac{\mp \frac{i}{4}\left(4 \lambda t^{2} C_{2}{ }^{2}+8 \lambda t C_{2} C_{3}-C_{2}{ }^{2} r^{2} \mp 4 C_{1} C_{2} \sqrt{t^{2} C_{2}{ }^{2}+2 t C_{2} C_{3}+C_{3}^{2}-4}+4 \lambda C_{3}{ }^{2}-16 \lambda\right)}{C_{2} \sqrt{t^{2} C_{2}{ }^{2}+2 t C_{2} C_{3}+C_{3}{ }^{2}-4}}} \\
& \cdot \frac{1}{2}\left(C_{2} t \mp \sqrt{t^{2} C_{2}^{2}+2 t C_{2} C_{3}+C_{3}{ }^{2}-4}+C_{3}\right),
\end{aligned}
$$

where $C_{1}, C_{2}$ and $C_{3}$ are the same constants in (12).

By (13), it is not difficult to find out $C_{1}=0$ and $C_{2}=C_{3}=2$ derive the solution as the following form

$$
U=\mathrm{e}^{\frac{-i\left(4 \lambda t^{2}+8 t \lambda-r^{2}\right)}{4 \sqrt{t(t+2)}}}(t-\sqrt{t(t+2)}+1) .
$$


In this situation, we can use (7) to obtain the solution of (1) as follows

$$
\left(s_{1}, s_{2}, s_{3}\right)=\left( \pm \frac{\cos \frac{-\left(4 \lambda t^{2}+8 t \lambda-r^{2}\right)}{4 \sqrt{t(t+2)}}}{\sqrt{t(t+2)}}, \pm \frac{\sin \frac{-\left(4 \lambda t^{2}+8 t \lambda-r^{2}\right)}{4 \sqrt{t(t+2)}}}{\sqrt{t(t+2)}}, \frac{t+1}{\sqrt{t(t+2)}}\right) .
$$

\section{Finite Time Singularity Solution II}

In this section, we construct the solution from another point of view. Considering the equation under the two-dimensional cylindrical coordinates, (1) adopts the following form

$$
S_{t}=S \dot{\wedge}\left(S_{r r}+\frac{u_{r}}{r}\right)+S \dot{\wedge}(S L) .
$$

In the two-dimensional space, we assume that the solution of (16) is as the following form

$$
\left\{\begin{array}{l}
s_{1}(t, r)=\cos (M(t, r)) F(t), \\
s_{2}(t, r)=\sin (M(t, r)) F(t), \\
s_{3}(t)=-\sqrt{1+(F(t))^{2}},
\end{array}\right.
$$

where $M(t, r)$ and $F(t)$ are the functions to be determined.

By (17), (16) is simplified into the differential equations of $M(t, r)$ and $F(t)$

$$
\left\{\begin{array}{l}
-\frac{\sqrt{1+(F(t))^{2}}\left(\frac{\partial}{\partial r} M(t, r)\right) F(t)}{r}-F(t)\left(\frac{\partial^{2}}{\partial r^{2}} M(t, r)\right) \sqrt{1+(F(t))^{2}} \\
-\frac{\mathrm{d}}{\mathrm{d} t} F(t)=0 \\
F(t) \sqrt{1+(F(t))^{2}}\left(\frac{\partial}{\partial r} M(t, r)\right)^{2}+F(t) \sqrt{1+(F(t))^{2}} \lambda \\
+F(t) \frac{\partial}{\partial t} M(t, r)=0 .
\end{array}\right.
$$

Furthermore, we set $M(t, r)=G(t) P(r)+Q(t)$. By (18), we obtain the following equations

$$
\left\{\begin{array}{l}
-\frac{\sqrt{1+(F(t))^{2}} G(t)\left(\frac{\mathrm{d}}{\mathrm{d} r} P(r)\right) F(t)}{r}-F(t) G(t) \sqrt{1+(F(t))^{2}} \frac{\mathrm{d}^{2}}{\mathrm{~d} r^{2}} P(r) \\
-\frac{\mathrm{d}}{\mathrm{d} t} F(t)=0 \\
F(t) \sqrt{1+(F(t))^{2}}(G(t))^{2}\left(\frac{\mathrm{d}}{\mathrm{d} r} P(r)\right)^{2}+F(t) \sqrt{1+(F(t))^{2}} \lambda \\
+F(t)\left(\frac{\mathrm{d}}{\mathrm{d} t} G(t)\right) P(r)+F(t) \frac{\mathrm{d}}{\mathrm{d} t} Q(t)=0 .
\end{array}\right.
$$


Setting $P(r)=\frac{1}{4} r^{2},(19)$ can be simplified as

$$
\left\{\begin{array}{l}
\sqrt{1+(F(t))^{2}} G(t) F(t)+\frac{\mathrm{d}}{\mathrm{d} t} F(t)=0, \\
F(t) \sqrt{1+(F(t))^{2}} \lambda+F(t) \frac{\mathrm{d}}{\mathrm{d} t} Q(t)=0,
\end{array}\right.
$$

Resolving (20), we find out that

$$
\left\{\begin{array}{l}
F(t)= \pm \frac{1}{\sqrt{t^{2} C_{1}^{2}+2 t C_{1} C_{2}+C_{2}^{2}-1}}, \\
Q(t)=-\sqrt{t^{2} C_{1}^{2}+2 t C_{1} C_{2}+C_{2}^{2}-1} \operatorname{sign}\left(t C_{1}+C_{2}\right) \lambda C_{1}^{-1}, \\
G(t)=\frac{\operatorname{sign}\left(t C_{1}+C_{2}\right) C_{1}}{\sqrt{t^{2} C_{1}^{2}+2 t C_{1} C_{2}+C_{2}^{2}-1}} .
\end{array}\right.
$$

Combining (21) and (17), (16) ensures the following solution

$$
\left\{\begin{aligned}
s_{1}(t, r)= & \cos \left(\frac{\operatorname{sign}\left(t C_{1}+C_{2}\right)\left(4 \lambda t^{2} C_{1}^{2}+8 \lambda t C_{1} C_{2}-C_{1}^{2} r^{2}+4 \lambda C_{2}^{2}-4 \lambda\right)}{4 C_{1} \sqrt{t^{2} C_{1}^{2}+2 t C_{1} C_{2}+C_{2}^{2}-1}}\right) \\
& \cdot \frac{ \pm 1}{\sqrt{t^{2} C_{1}^{2}+2 t C_{1} C_{2}+C_{2}^{2}-1}}, \\
s_{2}(t, r)= & \sin \left(\frac{\operatorname{sign}\left(t C_{1}+C_{2}\right)\left(4 \lambda t^{2} C_{1}^{2}+8 \lambda t C_{1} C_{2}-C_{1}^{2} r^{2}+4 \lambda C_{2}^{2}-4 \lambda\right)}{4 C_{1} \sqrt{t^{2} C_{1}^{2}+2 t C_{1} C_{2}+C_{2}^{2}-1}}\right) \\
& \cdot \frac{ \pm 1}{\sqrt{t^{2} C_{1}^{2}+2 t C_{1} C_{2}+C_{2}^{2}-1}}, \\
s_{3}(t)= & \frac{\left|t C_{1}+C_{2}\right|}{\sqrt{t^{2} C_{1}^{2}+2 t C_{1} C_{2}+C_{2}{ }^{2}-1}} .
\end{aligned}\right.
$$

where $C_{1}, C_{2}$ and $C_{3}$ are constants.

Selecting $C_{1}=C_{2}=1$, (22) can be simplified into

$$
\left(s_{1}, s_{2}, s_{3}\right)=\left( \pm \frac{\cos \frac{4 \lambda t^{2}+8 t \lambda-r^{2}}{4 \sqrt{t(t+2)}}}{\sqrt{t(t+2)}}, \pm \frac{\sin \frac{4 \lambda t^{2}+8 t \lambda-r^{2}}{4 \sqrt{t(t+2)}}}{\sqrt{t(t+2)}}, \frac{t+1}{\sqrt{t(t+2)}}\right) .
$$

There are only subtle differences between (23) and (15). In fact, if we take $P(r)=-\frac{1}{4} r^{2}$, similar to the above deducing procession, we get

$$
\left\{\begin{array}{l}
F(t)= \pm \frac{1}{\sqrt{t^{2} C_{1}^{2}+2 t C_{1} C_{2}+C_{2}^{2}-1}}, \\
Q(t)=-\sqrt{t^{2} C_{1}^{2}+2 t C_{1} C_{2}+C_{2}^{2}-1} \operatorname{sign}\left(t C_{1}+C_{2}\right) \lambda C_{1}^{-1}, \\
G(t)=-\frac{\operatorname{sign}\left(t C_{1}+C_{2}\right) C_{1}}{\sqrt{t^{2} C_{1}^{2}+2 t C_{1} C_{2}+C_{2}^{2}-1}},
\end{array}\right.
$$


According to (24), A solution similar to (22) can be found out.

\section{Conclusions}

Obviously, solution (23) (or (15)) forms a singularity in $t=0$. In fact, (22) covers the different types solutions. This solution can be a global solution(or a local solution) about time variables. If the constants are selected appropriately, the norm of gradient can form a singularity in finite time. Of course, the norm will not become infinity if we choose some special constants. Exactly, we obtain

$$
\left|S_{r}\right|^{2}=(F(t))^{2}\left(\frac{\partial}{\partial r} M(t, r)\right)^{2}=\frac{C_{1}^{2}\left(\operatorname{sign}\left(t C_{1}+C_{2}\right)\right)^{2} r^{2}}{4\left(t^{2} C_{1}^{2}+2 t C_{1} C_{2}+C_{2}^{2}-1\right)^{2}} .
$$

(23) is a function that has nothing to do with $\lambda$; Obviously, if $-\frac{C_{2} \pm 1}{C_{1}} \geq 0$, (23) will form a singularity at $t=-\frac{C_{2} \pm 1}{C_{1}}$. However, if $-\frac{C_{2} \pm 1}{C_{1}} \leq 0,(23)$ is a global solution. Exactly, if we set $C_{1}=1$ and $C_{2}=-2$, the exact form of the solution is

$$
\left\{\begin{array}{l}
s_{1}(t, r)=\frac{1}{\sqrt{t^{2}-4 t+3}} \cos \left(\frac{\operatorname{sign}(t-2)\left(4 \lambda t^{2}-16 \lambda t-r^{2}+12 \lambda\right)}{4 \sqrt{t^{2}-4 t+3}}\right), \\
s_{2}(t, r)=\frac{1}{\sqrt{t^{2}-4 t+3}} \sin \left(\frac{\operatorname{sign}(t-2)\left(4 \lambda t^{2}-16 \lambda t-r^{2}+12 \lambda\right)}{4 \sqrt{t^{2}-4 t+3}}\right), \\
s_{3}(t)=\frac{|t-2|}{\sqrt{t^{2}-4 t+3}}
\end{array}\right.
$$

(26) will form the singularity at $t=1$ and $t=3$. However, we just need to adjust the coefficient $C_{1}=1$ and $C_{2}=-2$ to obtain a global smooth solution

$$
\left\{\begin{array}{l}
s_{1}(t, r)=\frac{1}{\sqrt{t^{2}+4 t+3}} \cos \left(\frac{\operatorname{sign}(t+2)\left(4 \lambda t^{2}+16 \lambda t-r^{2}+12 \lambda\right)}{4 \sqrt{t^{2}+4 t+3}}\right), \\
s_{2}(t, r)=\frac{1}{\sqrt{t^{2}+4 t+3}} \sin \left(\frac{\operatorname{sign}(t+2)\left(4 \lambda t^{2}+16 \lambda t-r^{2}+12 \lambda\right)}{4 \sqrt{t^{2}+4 t+3}}\right), \\
s_{3}(t)=\frac{|t+2|}{\sqrt{t^{2}+4 t+3}} .
\end{array}\right.
$$

In fact, we can see that (27) is a global smooth solutions of (1) while (26) is a singularity one. It will be clear that given smooth initial data as in SME 
does a smooth initial data can develop two different behavior. Furthermore, the energy of the solution in section 3 can be a finite value on the finite spacial domain in its initial time. In $[7,8]$, although the solution is similar to (26) (or (27)), the solution of LLE is only a smooth one on $S^{2}$. In another words, $\mathcal{H}^{2}$ will lead to a singularity while the $S^{2}$ will not. In fact, the manifold affects the nature of the nonlinear term of the equation. Hence, the geometry of the target manifold plays an important role in the equation.

\section{Acknowledgments}

The author is grateful to the seminar about the blowup problem of Schrödinger map equation which was held in the school of Academy of Mathematics and Systems Science. This work is supported by NSFC (No.11161057), National Science Foundation for Young Scientists of China (No.11301014), TianYuan Special Funds of the National Natural Science Foundation of China (No.11426068), Project for Young Creative Talents of Ordinary University of Guangdong Province (No.XM060013) and Appropriative Researching Fund for Professors and Doctors of Guangdong University of Education (No. 2013ARF01).

\section{References}

[1] Q. Ding, Explicit blow-up solutions to the Schrödinger maps from $R^{2}$ to the hyperbolic 2-space $\mathcal{H}^{2}$, J. Math. Phys., 50, No. 10 (2009), 103507, 17 pages, doi: $10.1063 / 1.3218848$.

[2] S.J. Ding, C.Y. Wang, Finite time singularity of the Landau-LifshitzGilbert equation, Int. Math. Res. Not., 4 (2007), 25 pages, doi: 10.1093/imrn/rnm012.

[3] B.L. Guo, Y.Q. Han, G.S. Yang, Blow up problem for Landau-Lifshitz Equations in Two Dimensions, Commun. Nonlinear Sci. Numer. Simul., 5, No. 1 (2000), 43-44, doi: 10.1016/S1007-5704(00)90023-6.

[4] H. Huh, Blow-up solutions of modified Schrödinger maps, Comm. Partial Differential Equations, 33, No. 2 (2008), 235-243, doi: $10.1080 / 03605300701588748$.

[5] F. Merle, P. Raphäl, I. Radnianski, Blowup dynamics for smooth data equivariant solutions to the critical Schrödinger map problem, Invent. Math., 193, No. 2 (2013), 249-365, doi: 10.1007/s00222-012-0427-y. 
[6] J.B. Van Den Berg, J.F. Williams, (In-)stability of singular equivariant solutions to the Landau-Lifshitz-Gilbert equation, Eur. J. Applied Math., 24, No. 6 (2013), 921-948, doi: 10.1017/S0956792513000247.

[7] G.S. Yang, X.G. Liu, Spherical cone symmetric families generated by Landau-Lifshitz equation and their evolution, Sci. Sin. Math., 41, No. 2 (2011), 181-196, doi: 10.1360/012010-582.

[8] G.S. Yang, B.L. Guo, Some exact solutions to multidimensional LandauLifshitz equation with uprush external field and anisotropy field, Nonlinear Anal., 71, No. 9 (2009), 3999-4006, doi: 10.1016/j.na.2009.02.070.

[9] P.H. Zhong, S. Wang, S.T. Chen, Some periodic and blow-up solutions for Landau-Lifshitz equation, Mod. Phys. Lett. A, 26, No. 32 (2011), 24372452, doi: 10.1142/S0217732311036644.

[10] P.H. Zhong, S. Wang, M. Zeng, Some exact blowup solutions to multidimensional Schrödinger map equation on hyperbolic space and cone, Modern Phys. Lett. A, 28, No. 10 (2013), 1350043, 13 pages, doi: 10.1142/S0217732313500430

[11] P.H. Zhong, S. Wang, M. Zeng, Two blowup solutions for the inhomogeneous isotropic Landau-Lifshitz equation, J. Math. Anal. Appl., 409, No. 1 (2014), 74-83, doi: 10.1016/j.jmaa.2013.06.061. 
